\title{
Facelift surgery : the importance of incision design and scar prevention
}

\author{
Tran Nguyen Giap, MD' ${ }^{1}$, Albert Fox, MD, FACS ${ }^{2}$ \\ 'Department of Plastic and Reconstructive Surgery, University of Medicine Pham Ngoc Thach, Ho Chi Minh, Vietnam \\ ${ }^{2}$ AF Facial Plastic Surgery Center, Dartmouth, MA, United States
}

At the time of writing this article, I have performed $300+$ facelifts, with the following report: $91.0 \%$ female ( 273 cases), $9.0 \%$ male ( 27 cases). Traditional facelifts with pre- and postauricular flaps were $89.2 \%$ of cases, $8.0 \%$ were traditional comprehensive facelifts with a neck lift (midline platysmaplasty), and $2.8 \%$ were short scar facelifts. The operative reports and before and after photos for these cases were reviewed to evaluate incision placement technique and design. The goal of this case series report is to convey the importance of incision placement and scar prevention in facelift surgery.

Keywords: facelift; necklift; scar prevention

\section{Introduction}

Our face gives us our identity, helps express emotions, and affects our self-esteem and interactions with others. The smallest change in appearance can result in embarrassment and loss of confidence or can create a negative impression from others. A facelift is a powerful surgery that affects the physical appearance and psychological well-being of patients.

With other plastic surgery procedures, scars and poor results can be hidden under clothing; however, poor results and scarring on the face are exposed and can be seen. Because of this, no other aesthetic surgery carries the physical and emotional impact of a well-done facelift. Any resulting unnatural anatomy or a bad scar may cause a lifetime of problems that are impossible to hide.

The last several years have seen significant growth in facial aesthetic surgeries. With a deeper understanding of facial anatomy and the aging process, many techniques have been pub- lished and described (e.g., deep plane technique, SMASectomy, MACS lift) [1-8]. However, in the years to follow surgery, patients will not remember the technique that was used; they will

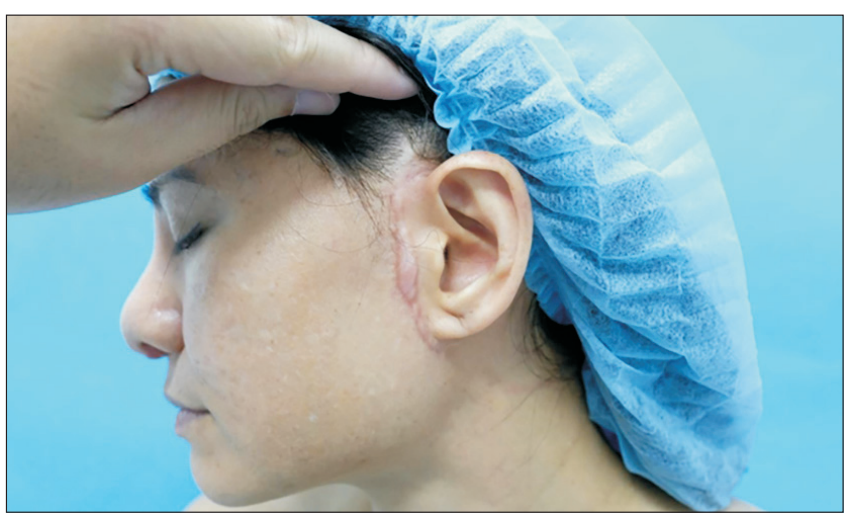

Fig. 1. The only lifetime reminder of facelift surgery is the resulting scar. Poor incision design will not only cause a bad scar but can affect other important areas including the earlobe, the hairline, and the tragus.

Received November 7, 2019; Revised December 30, 2019; Accepted December 30, 2019 
only see the incisions and scars that remain. This is especially true for Asian patients who have thicker skin and are prone to poor scarring (Fig. 1).

\section{Incision design}

While many surgeries are about what happens under the incision, most of the success or failure of facelift surgery revolves around how the incisions were designed.

Poor incision design can result in unnatural and abnormal anatomic changes such as misalignment of the hairline, loss of the temporal hair tuft, hair loss, scarring, tragal malposition, and pixie ear deformity (Fig. 2B).

Precise incision design in cosmetic surgery is necessary to avoid such complications and the stigma of having an "operated-on" look. Our goal as aesthetic surgeons is to make the incisions as inconspicuous as possible and give patients a natural appearance (Fig. 2A).

There are many ways to design incisions. These designs often vary in male and female patients. I have reviewed my facelift patient case series and describe the techniques and incision designs that have produced favorable aesthetic results.

It is important when creating incisions, that the patient is positioned upright. Carefully evaluate the position of the hairline, temporal tuft, ear lobe, and tragus. Evaluate the anatomical landmarks and position of natural creases and rhytids in relation to where incisions can be placed to maximize their camou-
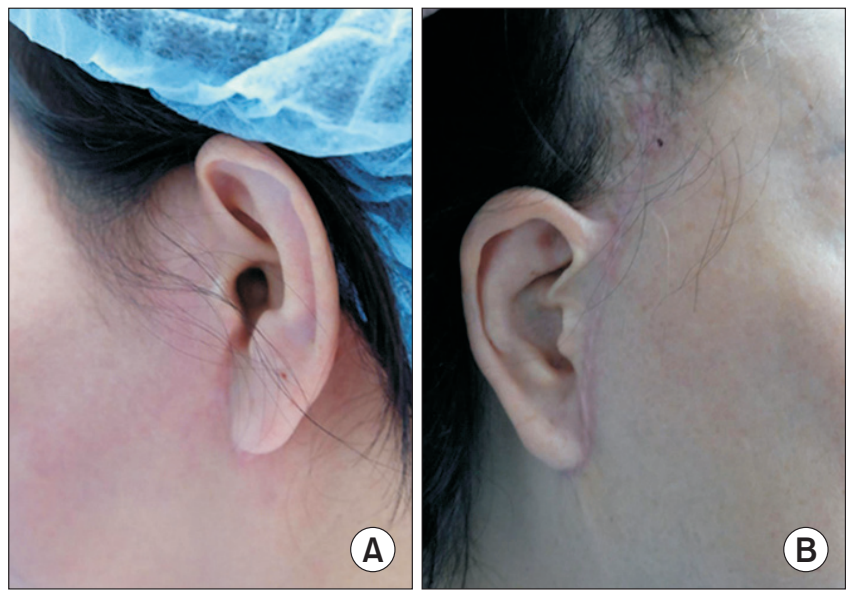

Fig. 2. (A) A good incision design. After 2 years, it's difficult to see the scar and appears natural. (B) This patient was operated by another surgeon and demonstrates many problems with design. A straight-line incision extends above the ear into the temporal hairline. This results in elevation of the sideburn and loss of the temporal tuft with resection of skin. This results in a very unnatural appearance. flage.

Anatomy to carefully evaluate:

- Skin quality and elasticity

· Hair coverage, hair loss (e.g., thinning hair)

- Position of sideburns and the temporal hair tuft

- Tragus shape, size, and position

- Ear lobe shape, size, and attachment to the cheek

- Natural creases or rhytids

- Submental crease position, length, and depth

Female and male incision designs have different nuances. Incision planning and complications of poor incision placement will be reviewed.

\section{Frontal and temporal areas}

Poor incision design can result in an unnatural hairline or even hair loss at the temporal tuft. In the frontal and temporal regions, it is important to avoid changing the hairline as much as possible. Elevation of the hairline and temporal tuft superior to the level of the root of the helix will appear abnormal and contribute to the stigma of an "operated-on" look. Unfortunately, many examples of sideburn elevation and loss of temporal hair tuft exist.

Often, physicians place an incision along the hairline to prevent temporal tuft elevation. However, there is a risk of forming a visible scar. If incision placement is along the hairline, it is important to make a trichophytic incision with a beveled scalpel to minimize scarring. By holding the scalpel at a 20- to 45-degree angle, the transected hair follicles will regrow through the scar.

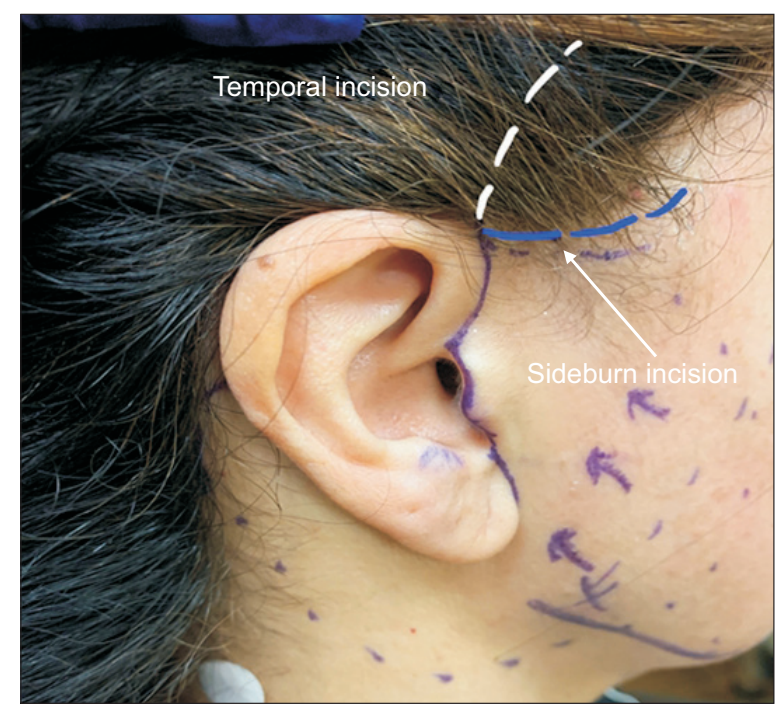

Fig. 3. It is necessary to excise a triangle of skin just below the temporal sideburn at the level of the superior root of the helix. 
The incision will be hidden by hair regrowth and, thus, will be less noticeable.

When there is minimal temporal hairline shift, the preferred incision is well within the temporal hair region. It is very reasonable to place the incision within the temporal hair tuft where the incision would be surrounded by hair. At times, it will be necessary to excise a triangle of skin just below the temporal sideburn at the level of the superior root of the helix (Fig. 3). This technique will remove excess skin and prevent an upward shift of the sideburn.

\section{Helical rim}

The color and texture of the skin on the ear and face are often different. This demarcation can contribute to more visible scarring. Improper incision placement at the helical root can result in a very noticeable scar. If the incision is placed too anterior to the helical root, the incision can be obvious. If the incision is too close to the helical root, the hair-bearing skin can be positioned unnaturally close to the ear or onto the ear itself. With the availability of laser hair removal, this can be less of a problem, but should, nonetheless, be avoided.

The surgeon should avoid drawing a straight line from the helical rim to the ear lobe (Fig. 4A). The use of curvilinear incisions or a series of crescent-shaped incisions around the ear can help minimize visible scarring. A good incision design should never involve a straight line, but rather consist of three distinct crescents around the ear. The first crescent should be around the root of the helix, the second around the tragus (anterior to the tragus or along the edge of the tragus), and the third around the ear lobe (Fig. 4B).

\section{Tragus}

Improper tragal incision design can result in a blunted tragus, anterior scarring of the tragus resulting in considerable visibility of the ear canal, or an obvious pre-tragal scar (Fig. 4C). Many patients are not aware of these tragal deformities; however, they can be noticed by friends and family. Other physicians will see these deformities as the surgeon's deficiency. There are two ways of addressing the tragus: a retrotragal (endaural) incision or a pretragal (preauricular) incision. There are advantages and disadvantages to both. In male patients, a pretragal incision is appropriate for numerous reasons. First, many males have a natural preauricular crease, which is convenient to hide an incision. Second, a pretragal incision in men can help to prevent
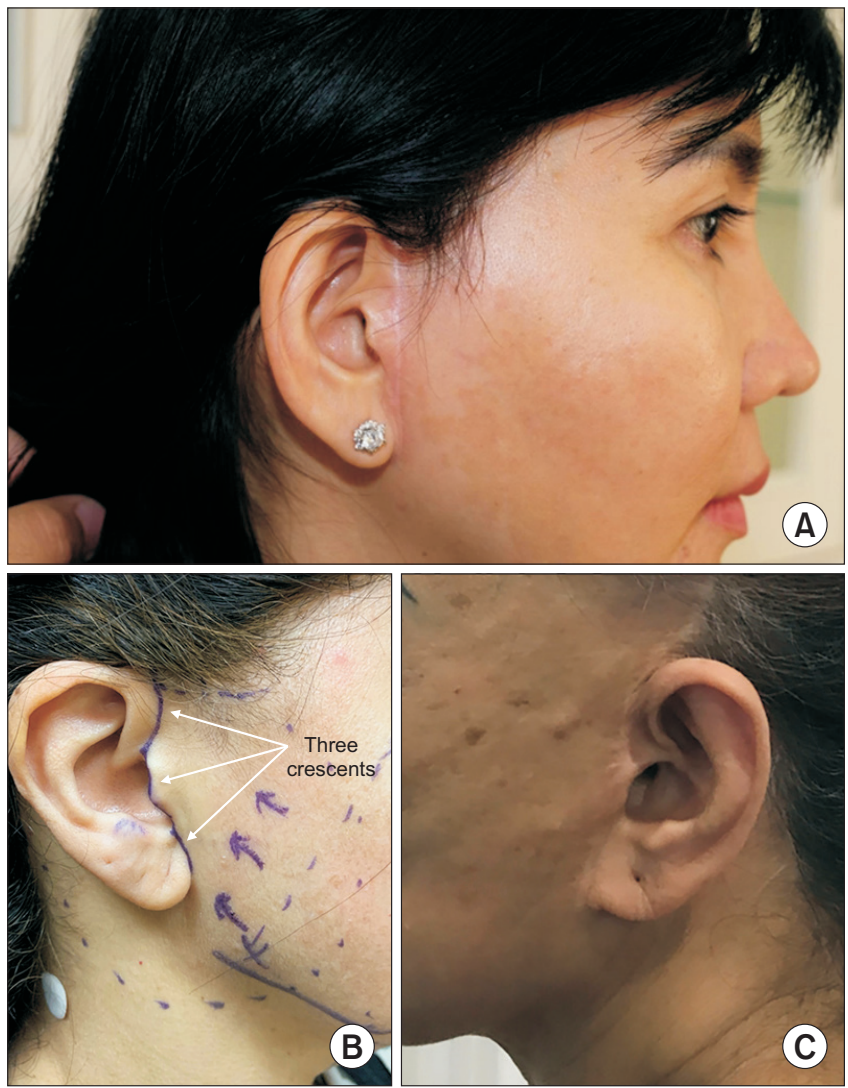

Fig. 4. (A) The straight incision should be avoided by the aesthetic surgeon. Even the healing is very optimal, the scar still can be very noticeable. (B) The first crescent around the root of the helix, the second around the tragus (anterior to tragus or along the edge of the tragus) and the third around the ear lobule. (C) A blunted tragus.

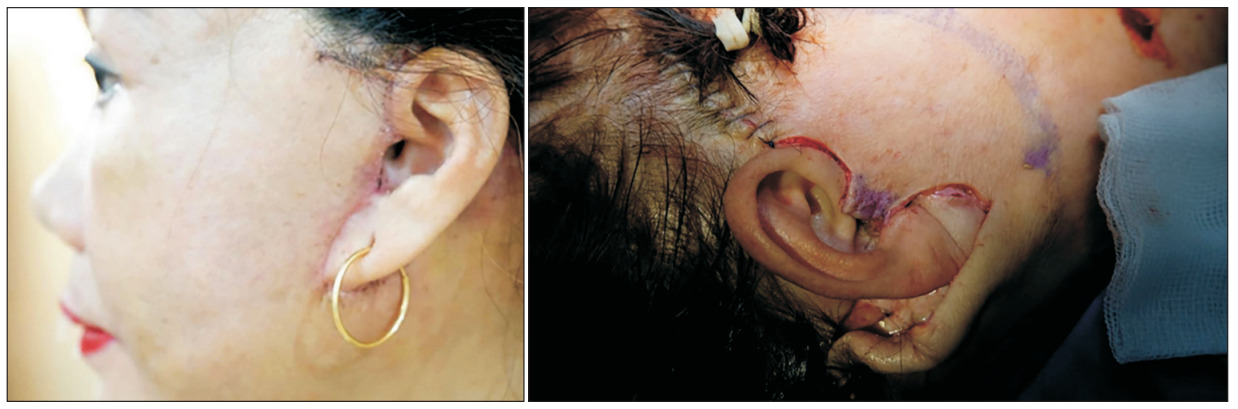

Fig. 5. After located another segment, I always address the tragus at last. Leaving no tension in this area is very important to prevent the blunted tragus or visible scar. The new tragus can be defined by carefully remove subcutaneous fat. 
the placement of bearded skin onto the tragus (Fig. 5).

In female patients, the same pretragal incision can be made; however, in my opinion, the retrotragal incision is preferred. A natural appearing tragus with minimal scarring can be created by mastering tragal flap contouring and reconstruction.

After contouring the flaps, I always address the tragus last. It is very important to leave no tension in this area to prevent a blunted tragus or visible scar. The new tragus can be defined by carefully removing subcutaneous fat (Fig. 5).

\section{Ear lobe}

Pixie ear deformity can occur after a facelift. This type of scarring is due to significant tension at the junction of the ear lobe and cheek/neck skin. The combination of skin contracture and gravitational forces can pull the ear lobe inferiorly and give it an unnatural, stretched appearance (Fig. 6A).

A small V-shaped incision preserving the angle at the caudal end of the ear lobe can be helpful. After the facelift work was completed, 3.0 polypropylene suspension sutures (Ethicon, LLC, USA) were applied deep at the SMAS level to prevent tension.

There are numerous descriptions of various techniques describing the postauricular incision design. The goal is to create a minimally noticeable scar with a natural hairline.

The postauricular incision can be placed directly in the sulcus. This helps to hide the scar behind the ear.

Placing the postauricular incision extending from the sulcus to the hairline at the level of the greatest width of the pinna produces the shortest scar (Fig. 6B).

Placing the incision at an inferior position to the greatest width of the pinna results in a more visible scar.

Placing any incision at a hairline/skin junction is acceptable if it heals well (Fig. 6C, D). Once again, the beveled trichophytic technique is helpful. This will allow hair follicles to regrow through the incision. The length of the hairline incision de-
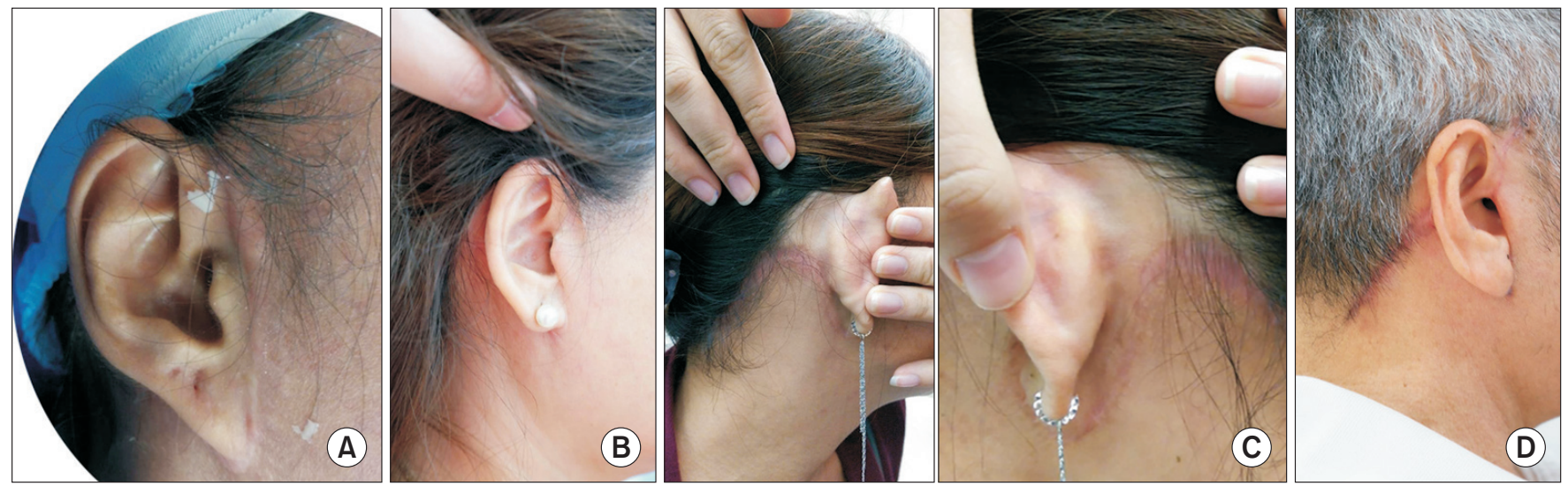

Fig. 6. (A) The combination of skin contracture and descent with gravity can pull the ear lobule inferiorly and give it an unnatural and stretched appearance. (B) The transverse hairline incision meet the post auricular at a 90 degree angle. (C) Although the cervical or occipital hairline incision is used by some surgeons, unfavorable scarring in this area is very visible and problematic. (D) A low mastoid incision is undesirable as there is more visible scar in that region.
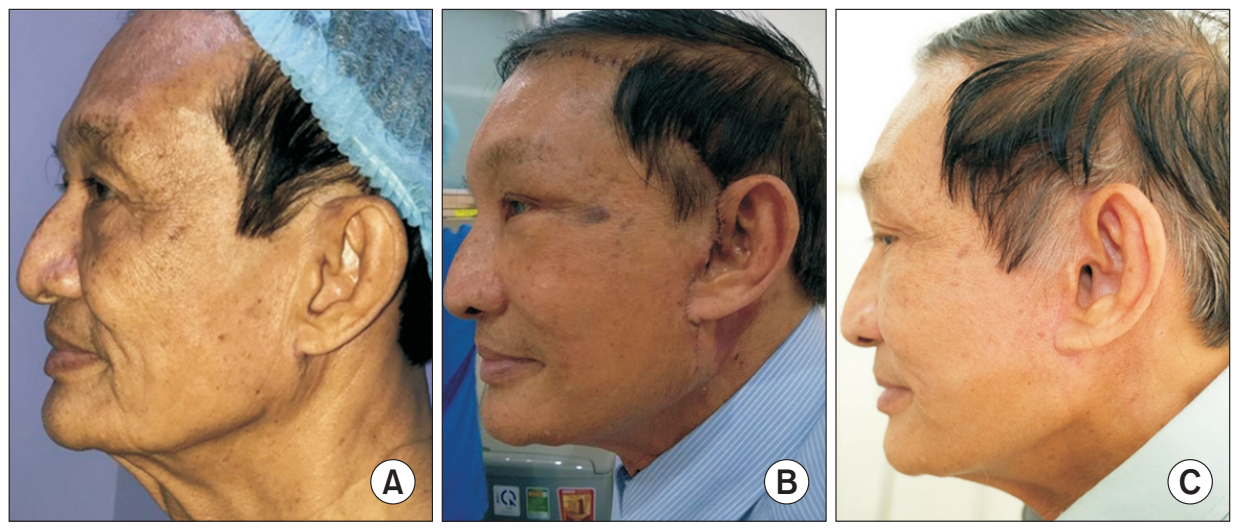

Fig. 7. Case report figures. (A) Before surgery, (B) two weeks after surgery, and (C) 12 months after surgery. 
pends on the patient's excess submandibular and cervical skin.

\section{Case report}

A 72-year-old male patient status post full facelift and forehead lift with a W-plasty incision at the forehead and three pretragal crescent incisions. After one year, the incisions healed well with minimal scarring (Fig. 7).

\section{Conclusion}

Favorable facelift results can be marred by poor scarring. The surgeon's skill is often judged by the appearance and quality of the final incisions. Good incision design, careful execution, and minimal tension on the wounds will often result in a natural appearance with a normal hairline position and minimal scarring. These are the hallmarks of a good facelift.

\section{Conflicts of interest}

The authors have nothing to disclose.

\section{References}

1. Niamtu J 3rd. Facelift surgery (cervicofacial rhytidectomy). In: Niamtu J 3rd, editor. Cosmetic facial surgery. New York: Elsevier; 2016. p. 247.

2. Baker DC, Nahai F, Massiha H, Tonnard P. Short scar face lift. Aesthet Surg J 2005;25:607-17.

3. Camirand A, Doucet J. A comparison between parallel hairline incisions and perpendicular incisions when performing a face lift. Plast Reconstr Surg 1997;99:10-5.

4. Baker DC. Complications of cervicofacial rhytidectomy. Clin Plast Surg 1983;10:543-62.

5. Baker DC. Minimal incision rhytidectomy (short scar face lift) with lateral SMASectomy: evolution and application. Aesthet Surg J 2001;21:14-26.

6. Stuzin JM, Baker TJ, Gordon HL, Baker TM. Extended SMAS dissection as an approach to midface rejuvenation. Clin Plast Surg 1995;22:295-311.

7. Baker TJ, Gordon HL, Stuzin JM, Peace D. Surgical rejuvenation of the face. 2nd ed. St. Louis (MO): Mosby; 1996.

8. Pina DP. Aesthetic and safety considerations in composite rhytidectomy: a review of 145 patients over a 3-year period. Plast Reconstr Surg 1997;99:670-8. 